\title{
8. Finanzplatz Schweiz
}

\section{Gérard Perroulaz}

\section{(2) OpenEdition \\ Journals}

Electronic version

URL: http://journals.openedition.org/sjep/266

DOI: $10.4000 /$ sjep.266

ISSN: 1663-9677

\section{Publisher}

Institut de hautes études internationales et du développement

\section{Printed version}

Date of publication: 1 avril 2003

Number of pages: 133-150

ISBN: 2-88247-050-3

ISSN: $1660-5926$

\section{Electronic reference}

Gérard Perroulaz, « 8. Finanzplatz Schweiz », Schweizerisches Jahrbuch für Entwicklungspolitik [Online], 22-1 | 2003, Online erschienen am: 04 Mai 2010, abgerufen am 08 September 2020. URL : http:// journals.openedition.org/sjep/266 ; DOI : https://doi.org/10.4000/sjep.266 


\section{FINANZPLATZ SCHWEIZ*}

EIT ANFANG DER 90ER JAHRE ist die schweizerische Gesetzgebung zur Bekämpfung der Geldwäscherei, des organisierten Verbrechens und der Korruption umfassend revidiert worden. Die Eidgenössische Bankenkommission zeigte sich besorgt über verschiedene Fälle von Potentatengeldern, die auf Schweizer Banken deponiert wurden (strenge interne Untersuchungen in den Fällen Abacha (Nigeria) und Montesinos (Peru). Der Bundesrat bewies seinen Willen, zu verhindern, dass der Finanzplatz Schweiz zur Terrorismusfinanzierung missbraucht wird (Blockierung von Guthaben im Einvernehmen mit den Vereinigten Staaten im Anschluss an den 11. September 2001). In Bezug auf Vermögenswerte, die aus Steuerfluchtgründen auf Schweizer Banken deponiert werden, nehmen sowohl die Bankenkreise als auch der Bundesrat eine defensive Haltung ein und betonen die Notwendigkeit, das schweizerische Bankgeheimnis aufrechtzuerhalten.

Im Rahmen der Überprüfung der schweizerischen Entwicklungszusammenarbeit (2000) begrüsste der Entwicklungshilfeausschuss der OECD (Development Assistance Committee, DAC) die in der Schweiz erzielten Fortschritte im Kampf gegen Korruption und Geldwäscherei. Im Hinblick auf die strafrechtlichen Bestimmungen der Schweiz bezüglich der Bestechung kritisierte die OECD allerdings die Tatsache, dass in der Schweiz die strafrechtliche Haftung von juristischen Personen nach wie vor nicht im Gesetz verankert ist. Nach Ansicht des DAC zeigt die Affäre um die Abacha-Gelder verschiedene potenzielle Kohärenzprobleme auf. Gelder verbrecherischer Herkunft seien durch das schweizerische Bankgeheimnis nicht geschützt, da dieses in Fällen von Korruption und Geldwäscherei sowie im Zusammenhang mit Potentatengeldern aufgehoben werde. Das Bankgeheimnis stelle also kein Hindernis für die internationale gerichtliche Zusammenarbeit auf diesem Gebiet dar. Da die Schweiz jedoch einen bedeutenden Finanzplatz beherberge, für den das Bankgeheimnis einen wichtigen Wettbewerbsfaktor darstellt, bestehe eine gewisse Gefahr, dass Vermögenswerte aus Entwicklungsländern mit zweifelhaftem Hintergrund angezogen werden. Aus diesem Grund müsse die Schweiz kontinuierlich sicherstellen, dass ihr Finanzplatz nicht missbraucht werde ${ }^{1}$.

\subsection{KAMPF GEGEN DIE GELDWÄSCHEREI}

- Internationale Tätigkeiten, OECD-Arbeitsgruppe gegen Geldwäscherei (FATF)

Die Arbeitsgruppe gegen Geldwäscherei (FATF) mit Sitz am OECD-Hauptquartier in Paris ist die internationale Koordinationsstelle für die Bekämpfung der

\footnotetext{
Von Gérard Perroulaz, Forschungsbeauftragter am iuéd.

CAD, Comité d'aide au développement, Examen en matière de coopération pour le développement, Suisse, dossiers du CAD 2000, Vol. 1, n 4, S. 52 und 53 „Cohérence et fuite de capitaux“.
} 
Geldwäscherei. 1990 hatte die FATF 40 Empfehlungen für eine effizientere Bekämpfung der Geldwäschereitätigkeiten herausgegeben, die mittlerweile als internationale Standards für Massnahmen in diesem Bereich anerkannt werden². Diese Empfehlungen besitzen zwar keinen bindenden Charakter, jedoch wurden mehrere Mechanismen erarbeitet, um den Kampf gegen derartige Machenschaften kontinuierlich fortzuführen: periodische gegenseitige Evaluierung der Gesetzgebung in den Mitgliedsstaaten, Seminare über neue Formen der Geldwäscherei, Veröffentlichung eines jährlichen Berichts ${ }^{3}$ und einer laufend aktualisierten Liste der nicht kooperativen Länder und Gebiete, Durchführung von Informationsveranstaltungen auch in Nichtmitgliedsländern.

Der jährliche Bericht über die Typologie der Geldwäschereitätigkeiten ${ }^{4}$ erörtert verschiedene Aspekte, die während der Periode 2001-2002 vertieft untersucht wurden. Insbesondere hat die FATF ihr Mandat einer Revision unterzogen und die Frage der Finanzierung terroristischer Aktivitäten (siehe unten) in ihren Tätigkeitsbereich integriert. Ferner befasste sich die FATF mit der Rolle der Korrespondenzbanken in der Geldwäscherei ${ }^{5}$ (Anfälligkeit und Möglichkeit zur Verschleierung der Herkunft von Geldern in den Geschäftsbeziehungen zwischen einer ausländischen und einer schweizerischen Bank), mit der Korruption und der Verwaltung von Privatvermögen (von Personen mit hohem Einkommen oder von Persönlichkeiten des öffentlichen Lebens).

2001 beschloss die FATF eine Überarbeitung ihrer 40 Empfehlungen. Im Mai 2002 wurde ein entsprechendes Dokument interessierten Kreisen zur Stellungnahme unterbreitet ${ }^{6}$. Die Revision der Empfehlungen dürfte nicht vor Mitte 2003 abgeschlossen sein. Im Zentrum steht eine Stärkung der Empfehlungen in drei Bereichen :

- Feststellung der Identität des Kunden und Sorgfaltspflicht, Meldung verdächtiger Transaktionen (mit besonderem Augenmerk auf „Risikokunden“);

๖ Feststellung der Identität der wirtschaftlich Berechtigten und der Personen, welche die Kontrolle über die einzelnen Gesellschaften ausüben (Unternehmen, Stiftungen, Treuhandunternehmen, Trusts);

- Möglichkeit einer Ausweitung des Geltungsbereichs der Empfehlungen auf den Nicht-Finanzsektor (Kasinos und weitere Spielbanken, Handel mit Immobilien und Luxusgütern, Unternehmensdienstleistungen, Rechtsanwälte, Notare, Buchhalter und Finanzberater).

Im Jahr 2000 hatte die FATF eine Liste der nicht kooperativen Länder und Gebiete veröffentlicht. Damit wollte sie diese Länder auffordern, verstärkt gegen die Geldwäscherei vorzugehen ${ }^{7}$. Diese Liste wird laufend aktualisiert und

2 Die Schweiz hält nahezu alle Empfehlungen der FATF ein. Allerdings hat die Schweiz das Übereinkommen der Vereinten Nationen gegen den illegalen Handel mit Betäubungsmitteln und psychotropen Stoffen noch nicht formell ratifiziert.

3 FATF, Financial Action Task Force on Money Laundering, Annual Report 2001-2002, 21 June 2002. Der Bericht ist auf der Website der FATF verfügbar.

4 GAFI, Rapport sur les typologies du blanchiment de capitaux 2001-2002, Paris, Februar 2002.

5 Eine Korrespondenzbank dient einem Bankinstitut auf einem anderen Finanzplatz, auf dem es nicht durch eine eigene Zweigstelle vertreten ist, als ständige Bankverbindung zur Abwicklung von Transaktionen. Quelle: Cotmec, La suisse dans la constellation des paradis fiscaux.

6 Verfügbar auf der Website unter : <www.fatf-gafi.org/pdf/Review40>.

7 Ein Bericht über nicht kooperative Länder und Gebiete findet sich auf folgender Website: <www. fatf-gafi.org/NCCT_fr.htm>. 2002 umfasste diese so genannte „schwarze Liste“ folgende Länder und 
umfasste im Juni 200215 als nicht kooperativ beurteilte Länder und Gebiete. Ein Land wird von der Liste gestrichen, sobald die Gesetzgebung ausreichend verstärkt wurde und die Verbesserungen in Kraft getreten sind. Gegen Länder, die keine wesentlichen Fortschritte vorweisen können, werden Sanktionen verhängt. Gegenwärtig sind beispielsweise gegen Nauru Sanktionen in Kraft, während die Ukraine und Nigeria ab 2003 mit Massnahmen rechnen müssen, sofern sie bis dahin keine ausreichenden Fortschritte erzielen. Nachdem Russland, Dominica, Niue und die Marshall-Inseln auf Grund ihrer Fortschritte bei der Bekämpfung der Geldwäscherei von der Liste gestrichen wurden, umfasst diese seit Oktober 2002 nur noch elf nicht kooperative Länder. Die Eidgenössische Bankenkommission rief die Schweizer Banken dazu auf, in ihren Beziehungen mit Personen, Unternehmen oder Banken aus nicht kooperativen Gebieten noch grössere Sorgfalt walten zu lassen. Besondere Vorsicht (minutiöse Kontrolle der Identität der Geschäftspartner und der wirtschaftlich Berechtigten) ist bei Finanztransaktionen mit Nauru geboten ${ }^{8}$.

\section{$\square$ Entwicklung des schweizerischen Finanzsektors}

Das von der G-7 ins Leben gerufene Forum für finanzielle Stabilität hatte im Jahr 2000 die Schweiz auf seine Liste der Offshore-Finanzplätze gesetzt ${ }^{9}$. Die Schweiz hatte entschieden gegen diesen Schritt protestiert und sich geweigert, sich den für Offshore-Finanzplätze vorgesehenen Kontrollen zu unterziehen. Im Gegenzug erklärte sie sich zu einer Evaluation ihres Finanzsektors durch das Financial Sector Assessment Programme (FSAP) des IWF bereit. Der abschliessende Bericht über diese Evaluation wurde im Juni 2002 veröffentlicht ${ }^{10}$. Im Zentrum des Berichts steht die Stärkung der Stabilität des Finanzsystems auf nationaler wie auf internationaler Ebene, aber auch die Geldwäschereiproblematik wird erörtert. Die Autoren gelangten zum Urteil, dass die für den Bankensektor geltenden schweizerischen Bestimmungen bezüglich der Geldwäscherei den internationalen Standards entsprechen. Hingegen kritisiert der Bericht, dass ausserhalb des Bankensektors (z.B. im Effektenhandel) die Identität des wirtschaftlich Berechtigten nicht systematisch überprüft wird.

\section{$\square$ Vollzug des schweizerischen Geldwäschereigesetzes}

Mit dem In-Kraft-Treten des neuen Geldwäschereigesetzes im April 1998 wurde der Geltungsbereich der einschlägigen Bestimmungen über den Bankensektor hinaus auf sämtliche Finanzintermediäre ausgeweitet. Die Überwachung des Systems obliegt der Eidgenössischen Finanzverwaltung. Die Finanzintermediäre müssen der MROS (Meldestelle für Geldwäscherei des Bundesamtes für Polizei) Meldung über verdächtige Transaktionen erstatten. Die MROS fungiert als

Gebiete: Ägypten, Cook-Inseln, Dominica, Grenada, Guatemala, Indonesien, Marshall-Inseln, Myanmar, Nauru, Nigeria, Niue, Philippinen, Russland, St. Vincent und die Grenadinen sowie die Ukraine.

8 Quelle: Mitteilung der EBK vom 7. Januar 2002 an die Banken, Effektenhändler und Fondsleitungen.

9 Die Liste umfasste 70 Offshore-Finanzplätze, darunter Luxemburg, Singapur und Hongkong. Hingegen wurden die bedeutenden Finanzplätze London, New York und Tokio nicht auf der Liste erwähnt. Das Forum für finanzielle Stabilität und die FATF definieren Offshore-Finanzplätze als Gerichtsbarkeit mit besonderen Merkmalen wie z.B. geringe Steuerbelastung, geringe Regelungsdichte und undurchdringbares Bankgeheimnis, die umfangreiche Aktivitäten aus dem Ausland anziehen und nur beschränkt mit anderen Ländern kooperieren.

10 Der Bericht ist auf folgender Website verfügbar : <www.imf.org/external/np/fsap/fsap.asp >. 
Drehscheibe zwischen dem Finanzsektor und den Strafverfolgungsbehörden. Sie untersucht die von den Finanzintermediären gemeldeten Fälle und leitet diese an die Justiz weiter, sofern sie sich als begründet erweisen. Die verdächtigen Guthaben bleiben während des ganzen Verfahrens blockiert.

Die Meldestelle für Geldwäscherei veröffentlicht jährliche Berichte über die Meldungen verdächtiger Transaktionen. In ihrem Tätigkeitsbericht $2001^{11}$ wies die MROS auf die markante Zunahme der gemeldeten verdächtigen Fälle hin (417 Fälle im Jahr 2001 gegenüber 311 im Jahr zuvor). 95 der 417 Meldungen erfolgten im Anschluss an die Anschläge vom 11. September bei der Suche nach Geldern, die mit der Finanzierung terroristischer Aktivitäten zusammenhängen. 91 Prozent aller Meldungen wurden an die Strafverfolgungsbehörden weitergeleitet. Dies ist als Beweis für die hohe Qualität der Meldungen zu werten. 61 Prozent der Meldungen stammen aus dem Bankensektor. Der Anteil der Meldungen seitens der Treuhänder, Finanzberater und Vermögensverwalter (insgesamt 29\%) ist in den vergangenen Jahren stetig angestiegen. Die Zahl der Meldungen von Rechtsanwälten, Notaren, Kasinos und Versicherungen ist sehr gering. 92 Prozent der gemeldeten Fälle stammen von Finanzintermediären aus den folgenden fünf Kantonen: Zürich, Genf, Bern, Tessin und Basel-Stadt. 31 Prozent der Meldungen über verdächtige Transaktionen wurden auf Grund von Informationen Dritter (von den USA im Anschluss an die Anschläge vom 11. September veröffentlichte Liste verdächtiger Namen) erstattet. In 28 Prozent der Fälle waren es in der Presse erschienene Nachrichten, welche die Finanzintermediäre veranlassten, den Hintergrund gewisser Kunden genauer zu überprüfen. In 14 Prozent der Fälle sind es die Kunden selbst, die den Verdacht des Finanzintermediärs wecken, weil sie den wirtschaftlichen Hintergrund einer Transaktion nicht schlüssig darlegen können. In weiteren 11 Prozent der Fälle machen die Strafverfolgungsbehörden die Banken auf Geschäftsbeziehungen aufmerksam, bei denen ein Zusammenhang mit anderen verdächtigen oder verurteilten Personen besteht.

\section{$\square$ Überarbeitung der Richtlinien der Eidgenössischen Bankenkommission zur Bekämpfung und Verhinderung der Geldwäscherei}

Die Eidgenössische Bankenkommission (EBK) rief eine Arbeitsgruppe ins Leben, die sich mit der Revision der EBK-Richtlinien zur Bekämpfung und Verhinderung der Geldwäscherei befassen sollte. Die Richtlinien aus dem Jahr 1998 präzisierten die Konsequenzen der Einführung des Geldwäschereigesetzes für die Schweizer Banken. Parallel dazu überarbeitete die Schweizerische Bankiervereinigung ihre Standesregeln zur Sorgfaltspflicht der Banken. Die so genannte Sorgfaltspflichtsvereinbarung, welche Regeln für die Überprüfung der Identität des Kunden umfasst, ist bereits seit 1977 in Kraft und wurde seither mehrmals überarbeitet, um den Neuerungen in der Gesetzgebung Rechnung zu tragen.

Der 2002 von der EBK in die Vernehmlassung geschickte Entwurf der Geldwäschereiverordnung sieht eine verstärkte Kontrolle zweifelhafter Transaktionen und eine Überwachung der Kundenbeziehungen vor. Dabei geht es nicht mehr nur darum, zweifelhafte Kunden zu erkennen, sondern sämtliche bestehenden Kundenbeziehungen mit Hilfe informatikgestützter Instrumente und Datenban-

11 MROS, 4. Jahresbericht 2001, Mai 2002. <www.bap.admin.ch>. 
ken im Detail zu durchleuchten, damit auffällige Transaktionen und Kunden noch besser ermittelt werden können. $\mathrm{Zu}$ den wichtigsten vorgeschlagenen Neuerungen zählt zweifellos der Vorschlag der EBK, wonach Finanzintermediäre ihre Geschäftsbeziehungen anhand der Höhe des Risikos in Kategorien einteilen müssen (Artikel 6 des Entwurfs der Geldwäschereiverordnung der EBK über die Sorgfaltspflichten der Banken und Effektenhändler). Für die Höhe des Risikos sind namentlich folgende Kriterien ausschlaggebend: Höhe der eingebrachten Vermögenswerte, Sitz oder Wohnsitz der Vertragspartei und des wirtschaftlich Berechtigten, Inkorporationsort von Gesellschaften, Art und Ort der Geschäftstätigkeit sowie Art der Konten oder der Geschäfte. Geschäftsbeziehungen mit politisch exponierten Personen (PEP) und solchen, die nicht zum üblichen Kundenkreis des Finanzintermediärs gehören, bergen ebenfalls ein erhöhtes Risiko (Artikel 16). Als Transaktionen mit erhöhten Risiken gelten solche, bei denen einmalig oder gestaffelt bedeutende Vermögenswerte (mehr als 100'000 Franken) eingebracht werden oder deren Betrag oder Häufigkeit auf Grund der bekannten Geschäftstätigkeit und der bekannten finanziellen Verhältnisse des Kunden als ungewöhnlich hoch erscheinen, sowie der Abzug bedeutender Beträge, die erst kurz zuvor eingebracht worden waren. Im Interesse des Rufes der Banken vertritt die EBK die Auffassung, dass diese sich nicht mit den Auskünften des Kunden oder seiner Vertreter begnügen dürfen, sondern dass sie diese anhand von Erkundigungen und öffentlich zugänglichen Quellen überprüfen und Transaktionen systematisch überwachen müssen, um ungewöhnliche Sachverhalte zu erkennen. Lehnt der Finanzintermediär eine Geschäftsbeziehung wegen begründeten Verdachts auf Geldwäscherei oder wegen eines Hinweises auf eine Verbindung mit Terrorismus ab, so muss er dies der MROS melden (damit geht Artikel 24 der Verordnung weiter als das Geldwäschereigesetz, welches lediglich für bestehende Geschäftsbeziehungen eine Meldepflicht vorsieht). Ferner muss der Finanzintermediär interne Weisungen erarbeiten und allen betroffenen Mitarbeitenden bekannt geben und dafür sorgen, dass die Mitarbeitenden hinsichtlich der für sie wesentlichen Aspekte der Geldwäschereibekämpfung dauernd ausgebildet werden. Finanzintermediäre müssen darüber hinaus über eine interne Geldwäschereifachstelle verfügen (Compliance-Organ) ${ }^{12}$, die jedoch die Geschäftsleitung und die Linienverantwortlichen nicht von ihrer Verantwortung befreit. Im Finanzbereich tätige Gruppengesellschaften und Banken mit ausländischen Zweigniederlassungen müssen die globale Überwachung der Reputationsrisiken im Zusammenhang mit Geldwäscherei und Terrorismusfinanzierung sicherstellen. Im Bedarfsfall müssen die internen Überwachungsorgane Zugang zu einer zentralen Datenbank der Vertragsparteien und der wirtschaftlich Berechtigten auf Gruppenebene haben. Die Verordnung der EBK gilt auch für ausländische Zweigniederlassungen von Schweizer Banken. In ihrem Fall erfolgt die Meldung verdächtiger Transaktionen oder allenfalls eine Vermögenssperre nach den Vorschriften des Gastlandes. Die neue Geldwäschereiverordnung der EBK tritt am 1. Juli 2003 in Kraft. Die darin enthaltenen Bestimmungen müssen ab Ende Juni 2004 vollumfänglich respektiert werden.

12 Die Fachstelle überwacht den Vollzug der internen Geldwäschereiweisungen und berät die Linienverantwortlichen in Fragen der Geldwäschereibekämpfung. 
In der Presse wurden verschiedene Stimmen laut, die auf die enormen Investitionen (Personal- und Informatikressourcen) hinweisen, die für eine umfassende Anwendung der vorgeschlagenen Weisungen erforderlich wären ${ }^{13}$.

Die Tätigkeit der Kundenberater im Anlagen- und Vermögensverwaltungsgeschäft ist alles andere als einfach. Neben der Anwerbung wohlhabender Kunden und der qualitativ hoch stehenden Beratung gilt es, gute Beziehungen mit bedeutenden Kunden zu pflegen, und zu verhindern, dass gewisse Kunden zu Konkurrenzbanken abwandern oder ihr Vermögen auf ausländischen Finanzplätzen anlegen. Gleichzeitig will das aktuelle Geschehen aufmerksam verfolgt werden, müssen Listen mit Namen verdächtiger Personen mit der Liste der Kunden verglichen und Kunden um Informationen gebeten werden. Nicht zuletzt aber muss verhindert werden, dass der Ruf des Finanzinstituts unter Affären mit Potentatengeldern leidet, über die in der Presse berichtet wird ${ }^{14}$.

\subsection{BUNDESGESETZ ÜBER DIE TEILUNG EINGEZOGENER VERMÖGENSWERTE}

Im Oktober 2001 legte der Bundesrat seine Botschaft betreffend das Bundesgesetz über die Teilung eingezogener Vermögenswerte vor ${ }^{15}$.

Deliktische Vermögenswerte werden zumeist nicht in dem Land beschlagnahmt, in dem das Verbrechen begangen wurde. Zudem sind an der Durchführung der entsprechenden Ermittlungen häufig mehrere Länder beteiligt. Die Idee der Teilung deliktischer Vermögenswerte wurde in den Vereinigten Staaten entwickelt (,asset sharing“). Der Grundsatz der Koordination der Strafverfolgung und der Teilung der Vermögenswerte ist Bestandteil der Empfehlungen der FATF, des Übereinkommens der Vereinten Nationen gegen den illegalen Handel mit Betäubungsmitteln und des Übereinkommens des Europarates von 1990 über Geldwäscherei sowie Ermittlung, Beschlagnahme und Einziehung von Erträgen aus Straftaten. In Fällen, in denen internationale Rechtshilfe gewährt wird, vereinbaren üblicherweise die beteiligten Länder die Regeln für die Aufteilung der fraglichen Vermögenswerte auf die einzelnen Staaten. Innerhalb der Schweiz sind die Regeln relativ diffus (Zuständigkeiten mehrerer Kantone im Rahmen eines Verfahrens und Einbezug des Bundes).

Die Frage nach der Teilung beschlagnahmter Werte stellt sich zum einen im internationalen (Aufteilung zwischen der Schweiz und anderen Staaten) und andererseits im nationalen Kontext (Aufteilung zwischen Bund und Kantonen). Der Entwurf regelt in erster Linie die internationale Zusammenarbeit und die Aufteilung der gesamten oder eines Teils der Vermögenswerte auf die am Verfahren beteiligten Länder. Ferner sieht der Entwurf ausdrücklich vor, dass Vermögenswerte, welche aus der Bestechung von ausländischen Beamten oder ungetreuer Amtsführung derselben stammen, dem „verletzten“ Staat vollumfänglich zurückerstattet werden können. Auf innerstaatlicher Ebene sieht der

Zum Beispiel Le Temps, 19. August 2002; NZZ.

14 Vgl. Dominique Froidevaux, „Un ,swiss private banker“ n'est pas un extraterrestre“, in La Suisse dans la constellation des paradis fiscaux, Ed. d'en Bas, 2002.

15 Botschaft betreffend das Bundesgesetz über die Teilung eingezogener Vermögenswerte vom 24. Oktober 2001, Bundesblatt 2002, 29.1.2002, S. 441-481 (Botschaft 01.064). 
Gesetzesentwurf vor, dass bei beschlagnahmten Vermögenswerten von über 100'000 Franken die Hälfte dem Gemeinwesen (Kanton oder Bund), welches das Verfahren geleitet und die Einziehung verfügt hat, zugeteilt wird. Der Kanton, in dem sich die deliktischen Vermögenswerte befinden, erhält als Vergütung für seine Mitarbeit im Verfahren 20 Prozent der Vermögenswerte. Die restlichen 30 Prozent sollen in jedem Fall dem Bund zufliessen, der diese für den Kampf gegen die Kriminalität einsetzt (internationale Rechtshilfe, zentrale Behörden, die an der Bekämpfung des organisierten Verbrechens beteiligt sind).

Mehrere Parlamentarier ${ }^{16}$ und NGO (z.B. die Arbeitsgemeinschaft der Hilfswerke) forderten, die aus Drogendelikten stammenden Gelder als Hilfe für Drogensüchtige (Prävention des Drogenmissbrauchs) oder zu Gunsten der Drogen produzierenden Länder (Förderung des Anbaus anderer Kulturen) zu verwenden. Wirtschaftsverbände und Strafverfolgungsbehörden haben sich im Zuge der Vernehmlassung gegen eine Zweckbindung der konfiszierten Gelder auf Kantonsebene ausgesprochen, während im Kampf gegen den Drogenmissbrauch tätige Kreise und NGO aus dem Bereich der Entwicklungszusammenarbeit die gezielte Verwendung der Gelder zur Förderung der Prävention im In- und Ausland befürworteten. In seiner Botschaft verzichtete der Bundesrat darauf, die Verwendung der beschlagnahmten Vermögenswerte im Detail zu regeln. Damit können die Kantone über den ihnen zustehenden Teil frei verfügen. Die Kantone Waadt, Genf und Freiburg sehen eine Verwendung der Mittel nach den oben erwähnten Grundsätzen vor. Im Kanton Genf sollen die aus dem Drogenhandel stammenden eingezogenen Vermögenswerte bis $\mathrm{zu}$ einer Höhe von maximal 3 Millionen Franken pro Jahr in einen speziellen Fonds eingezahlt werden. Die Mittel dieses Fonds sollen je zur Hälfte zur Drogenprävention im Kanton Genf und zur Finanzierung von Entwicklungshilfeprojekten verwendet werden $^{17}$. Im Kanton Freiburg können die beschlagnahmten Vermögenswerte auch zur medizinisch-sozialen Betreuung Drogenabhängiger verwendet werden, während der Kanton Waadt auch die Bekämpfung des Alkoholmissbrauchs als möglichen Verwendungszweck vorsieht. Seinen Entscheid, auf eine bundesrechtliche Zweckbindung zu verzichten und die Kantone über die Verwendung der ihnen zukommenden eingezogenen Vermögenswerte verfügen zu lassen, begründet der Bundesrat folgendermassen.

๖ Da die eingezogenen Vermögenswerte nicht allein aus dem Drogenhandel, sondern mehrheitlich aus anderen Straftaten stammen (z.B. Waffenhandel, Bestechung usw.), wäre es fragwürdig, diese Mittel ausschliesslich für die Bekämpfung des Drogenhandels einzusetzen.

$\checkmark$ Drogengelder werden oft durch komplizierte wirtschaftliche und finanzielle Transaktionen gewaschen. Angesichts dessen erscheint es legitim, diese Gelder zum Teil auch für die Finanzierung des Strafverfolgungsapparates zu verwenden.

๖ Die Schaffung eines besonderen Fonds widerliefe den finanzpolitischen Grundsätzen, wonach Bund und Kantonen bei der Verwendung ihrer Einnahmen ein gewisser Spielraum eingeräumt wird.

16 Motion Alex Heim und parlamentarische Initiative Jost Gross.

17 Loi genevoise du 26 mai 1994 sur la création d'un fonds destiné à la lutte contre la drogue et à la prévention de la toxicomanie. 


\subsection{SORGFALTSPFLICHTEN DER BANKEN UND EFFEKTENHÄNDLER GEGENÜBER POLITISCH EXPONIERTEN PERSONEN}

In die bedeutenden Affären von auf Schweizer Banken deponierten Fluchtgeldern, die seit dem Fall Marcos aus dem Jahr 1986 bekannt worden sind, waren häufig so genannte politisch exponierte Personen (PEP) verwickelt ${ }^{18}$. Die jüngsten Fälle dieser Art waren jene im Zusammenhang mit den Geldern des Abacha-Clans (Nigeria) und die Montesinos-Affäre (Peru).

Im Anschluss an die Abacha-Affäre fand auf Initiative der Schweiz im November 2000 eine Tagung von Vertretern von Justiz- und Bankenaufsichtsorganen der G-7-Staaten und der Schweiz statt. Ziel des Treffens war die Gegenüberstellung der je nach Land verschiedenen Systeme sowie die Erfassung der Vermögenswerte von Personen mit bedeutenden öffentlichen Aufgaben. Insbesondere sollten Wege gefunden werden, um zu verhindern, dass die in einem nationalen System nicht mehr geduldeten Vermögenswerte auf einen benachbarten Finanzplatz verschoben werden können ${ }^{19}$. Mehrere G-7-Staaten und die Schweiz entschlossen sich dazu, Empfehlungen zur Entgegennahme von Vermögenswerten solcher Personen zu verfassen. Diese Empfehlungen beziehen sich auf die erhöhten Sorgfaltspflichten der Banken in ihren Beziehungen mit Kunden dieser Kategorie. Die Banken müssen dabei auf gewisse Anzeichen achten, die eine erhöhte Aufmerksamkeit erfordern, wie z.B. Provisionszahlungen oder von einer Zentralbank eingehende Zahlungen. Ferner sind sie verpflichtet, ihre als PEP erkannten Kunden besonders zu überwachen und dabei speziell die öffentlich zugänglichen Quellen zu konsultieren und ihre Geschäftsbeziehungen mit solchen Personen regelmässig zu überprüfen. Der Entscheid, eine Person mit bedeutenden öffentlichen Aufgaben als Kunde zu akzeptieren, ist auf Direktionsebene zu fällen. Um festzustellen, ob eine Person dieser besonders zu überwachenden Kundenkategorie zuzuordnen ist, müssen namentlich die öffentlich zugänglichen Quellen konsultiert werden.

\section{$\square$ Affäre Montesinos (Peru)}

Die Eidgenössische Bankenkommission (EBK) hatte eine Untersuchung über das Verhalten der Banken bei der Entgegennahme von Geldern in der Abachaund Montesinos-Affäre durchgeführt. Im Fall der Korruptionsgelder, die der ehemalige peruanische Geheimdienstchef (und persönliche Berater von Ex-Präsident Fujimori) Vladimiro Lenin Montesinos Torres in der Schweiz deponiert hatte, führte die EBK ein aufsichtsrechtliches Verfahren gegen fünf Banken durch, um festzustellen, ob sie gegen die Sorgfalts- und Meldepflichten verstossen haben. Im Rahmen der Montesinos-Affäre wurden Guthaben in Höhe von 114 Millionen Franken aufgespürt und blockiert. Es handelte sich dabei um Gel-

18 Laut Definition der Eidgenössischen Bankenkommission (EBK) umfasst der Begriff ,politisch exponierte Personen“ Staatschefs, Regierungsmitglieder, hochrangige Vertreter der Justiz und des Militärs, Parlamentarier, hochrangige Polizei- und Behördenvertreter, oberste Organe staatlicher Unternehmen sowie Personen, welche diesen aus familiären oder anderen Gründen nahe stehen (Jahresbericht 2001 der EBK, S. 34 sowie Geldwäschereiverordnung der EBK). Eine Aufstellung der Affären, in die der Finanzplatz Schweiz verwickelt war, findet sich in G. Perroulaz, F. Membrez et D. Froidevaux, „La longue litanie des affaires“ in La Suisse dans la constellation des paradis fiscaux, Ed. d'en Bas, 2002.

19 Quelle: Jahresbericht 2000 der EBK, S. 22 ff., Jahresbericht 2001 der EBK, S. 34. 
der, die von Montesinos persönlich oder von ihm nahe stehenden Personen eingezahlt worden waren. Die Untersuchung der $\mathrm{EBK}^{20}$ förderte in einer der fünf Banken (Bank Leumi le-Israel, Schweiz) erhebliche Mängel zu Tage. Trotz der beträchtlichen Höhe der Vermögenswerte und des angegebenen Tätigkeitsgebietes (Waffenhandel), welche die Geschäftsleitung hätten alarmieren müssen, hatte es die Bank unterlassen, die wirtschaftlichen Hintergründe dieser Gelder zu überprüfen. Die EBK sah sich gezwungen, die Entfernung des Generaldirektors der Bank zu verlangen; die betreffende Bank akzeptierte diese Anordnung. Der Generaldirektor der Bank hatte die Kundenbeziehung zu Montesinos persönlich genehmigt und trug die Mitverantwortung dafür, dass die PEP-Eigenschaft von Montesinos nicht erkannt und die Herkunft der Gelder nicht überprüft wurde. Obwohl es erklärte Geschäftspolitik der Bank ist, keine PEP als Kunden anzunehmen, musste die EBK feststellen, dass die Bank kein Kontrollinstrument besass, um Kundenbeziehungen auf eine allfällige PEP-Eigenschaft hin zu überprüfen. Zudem wurden das ungeeignete Mahnwesen und die mangelhaften internen Weisungen beanstandet. Im August 2002 erhielt Peru im Rahmen des internationalen Rechtshilfeverfahrens 77,5 Millionen Dollar von der Schweiz zurückerstattet. Nach wie vor sind Guthaben in Höhe von 33 Millionen in der Schweiz eingefroren ${ }^{21}$. Die Untersuchung der Bezirksanwaltschaft des Kantons Zürich hatte ergeben, dass es sich dabei um Korruptionsgelder handelte (namentlich um „Kommissionszahlungen“ an Montesinos im Zusammenhang mit Waffenlieferungen nach Peru).

\section{$\square$ Kompromiss in der Affäre Abacha (Nigeria)?}

Die Affäre um die Gelder des Abacha-Clans wurde bekannt, nachdem die neuen gesetzlichen Bestimmungen betreffend die Meldepflicht bei verdächtigen Transaktionen bereits in Kraft getreten waren (Geldwäschereigesetz aus dem Jahr 1998). Im Anschluss daran reichte die nigerianische Regierung bei der Schweiz ein Gesuch um internationale Rechtshilfe ein.

Im April 2002 schloss die Regierung Nigerias mit den Angeklagten, die dem ehemaligen Präsidenten Sani Abacha (im Amt von 1993 bis 1998) nahe gestanden hatten, einen Vergleich ab, auf Grund dessen das Rechtshilfeverfahren - dessen Abschluss angesichts der zahlreichen Rekursmöglichkeiten noch nicht absehbar war - unter Umständen eingestellt werden könnte. Gemäss diesem Vergleich ${ }^{22}$ wird die nigerianische Regierung ihr Rechtshilfegesuch (und die Angeklagten ihre Rekurse) zurückziehen. Im Gegenzug werden in verschiedenen Ländern eingefrorene Gelder in Höhe von mehr als einer Milliarde Dollar (darunter 535 Millionen Dollar in der Schweiz ${ }^{23}$ ) an Nigeria ausbezahlt. Die Rückzahlung erfolgt über die Bank für Internationalen Zahlungsausgleich (BIZ, Basel), die zusammen mit der Weltbank über die Verwendung dieser Gelder entscheiden wird (z.B. zur Verringerung der Aussenverschuldung Nigerias). 100 Millionen Dollar werden an die Familienangehörigen Abachas ausbezahlt, die als Gegenleistung der Rückzahlung der übrigen Gelder zustimmen. Im Zusammenhang mit

20 Medienmitteilung der Eidgenössischen Bankenkommission, 13. November 2001.

21 Le Temps, 21.8.2002; NZZ.

22 Siehe Pressemitteilung der Bundesverwaltung vom 17. April 2002.

23 Bei den anderen beteiligten Ländern handelt es sich um Grossbritannien, Luxemburg, Liechtenstein und Jersey. 
dem Strafverfahren im Kanton Genf betreffend den Bruder Sani Abachas, der den Vergleich nicht unterzeichnet hat, bleiben Guthaben in Höhe von 90 Millionen Dollar auch weiterhin auf Schweizer Konten eingefroren.

Die Aktion Finanzplatz Schweiz äusserte Zweifel an der Rechtmässigkeit dieser aussergerichtlichen Einigung. Aus juristischer Sicht sind einige Aspekte unklar. Ferner kritisiert die NGO die Tatsache, dass die BIZ (die in ihren Entscheidungen unter dem Einfluss der Industrieländer steht) über die Verwendung der Gelder entscheiden soll, auf die der nigerianische Staat ein Anrecht hat. Ein möglicher Verwendungszweck ist die Verringerung der Aussenverschuldung Nigerias. Darüber hinaus hätten die Schweiz und Grossbritannien die nigerianische Kultur der Straffreiheit nicht unterstützen dürfen. Laut Ansicht der NGO zeigt die Notwendigkeit einer „gütlichen“ Einigung, dass Rechtshilfeverfahren nach wie vor durch zahlreiche Rekursmöglichkeiten in die Länge gezogen werden können. Im Dezember 2002 wurde das Strafverfahren wieder aufgenommen, da einige Mitglieder des Abacha-Clans die Einigung angefochten hatten. Das Rechtshilfeverfahren nimmt damit seinen Lauf und wird durch die Beschwerde der Familie Abacha gegen den Entscheid des Bundesamtes für Justiz, der nigerianischen Regierung Bankunterlagen zu übergeben, welche für das Verfahren in Nigeria von Nutzen sein könnten, erschwert.

\subsection{KAMPF GEGEN DAS ORGANISIERTE VERBRECHEN UND DIE TERRORISMUSFINANZIERUNG}

1999 hatte das Parlament einer Vorlage zugestimmt, wonach die Zuständigkeiten in Fällen von Geldwäscherei, organisiertem Verbrechen, Korruption und Wirtschaftskriminalität von den Kantons- auf die Bundesbehörden übertragen werden sollen. Die strafrechtliche Verfolgung in Fällen des organisierten Verbrechens, welche Verbindungen zum Ausland aufweisen, ist damit nicht mehr Sache der Kantone, sondern des Bundes. Diese Neuerung trat am 1. Januar 2002 in Kraft (Art. 340bis des Strafgesetzbuches).

Im Anschluss an die Anschläge vom 11. September 2001 in den Vereinigten Staaten war die öffentliche Debatte im Berichtsjahr durch einen Aspekt des organisierten Verbrechens massgeblich geprägt: den Terrorismus. Die Schweiz wollte frühzeitig den Beweis erbringen, dass sie das Risiko eines Missbrauchs bedeutender Finanzplätze für Operationen im Zusammenhang mit der Terrorismusfinanzierung durchaus ernst nimmt.

Die Geldwäscherei verfolgt das Ziel, die verbrecherische Herkunft von Geldern aus dem Drogenhandel und anderen illegalen Machenschaften zu verschleiern, indem schmutziges Geld in die üblichen Finanzkreisläufe eingeschleust wird. Seit den Terroranschlägen vom 11. September 2001 in den Vereinigten Staaten ist sich die Öffentlichkeit bewusst geworden, dass dieser Mechanismus auch umgekehrt funktioniert, indem „sauberes“ (aber auch schmutziges) Geld zur Finanzierung terroristischer Machenschaften verwendet wird. Die Gelder zur Finanzierung terroristischer Aktivitäten können aus der direkten finanziellen Unterstützung durch Staaten oder gewisse wohlhabende Personen oder aus anderen kriminellen Machenschaften stammen. Es kann sich aber auch um Gelder handeln, die auf rechtmässige Weise gesammelt wurden (legale wirtschaftli- 
che und Handelstätigkeiten, Sammelaktionen und Spenden, Beiträge, Verkauf von Publikationen, kulturelle oder gesellschaftliche Anlässe). Diese Gelder können entweder im Namen karitativer Organisationen oder durch verschiedene Unternehmen zusammengetragen werden. Die Methoden, die bei der Unterstützung terroristischer Aktivitäten zur Anwendung kommen, sind vergleichbar mit den klassischen Techniken des organisierten Verbrechens (Verschiebung illegal erworbener Vermögenswerte in Form von Bargeld, tranchenweise Einzahlungen und Abhebungen von Bankkonten, Kreditkarten usw.). Auch inoffizielle oder in gewissen Regionen verbreitete traditionelle Methoden (z.B. das HawalaSystem $^{24}$ ) erlauben es, Gelder zu verschieben.

Nach den Anschlägen vom 11. September forderten die Vereinigten Staaten und die Europäische Union Massnahmen, um Missbräuche des weltweiten Finanzsystems durch Terroristen besser aufdecken und verhindern zu können.

Im Oktober 2001 beschloss die FATF, ihr Tätigkeitsgebiet auf die Bekämpfung der Terrorismusfinanzierung auszuweiten. Es wurden acht spezifische Empfehlungen ${ }^{25}$ formuliert, welche folgende Aspekte abdecken: Anerkennung der Terrorismusfinanzierung und der Geldwäscherei im Rahmen terroristischer Aktivitäten als Straftatbestand; Blockierung und Beschlagnahmung von Vermögenswerten mit terroristischem Hintergrund; Verstärkung der Identifizierungsmassnahmen beim Transfer von Vermögenswerten; Meldung von Transaktionen, die vermutlich einen terroristischen Hintergrund haben; Überwachung von nicht gewinnorientierten Organisationen und juristischen Personen; elektronische Überweisungen sowie alternative Formen des Vermögenstransfers. Ferner empfiehlt die FATF den Staaten, das 1999 verabschiedete und im April 2002 in Kraft getretene Übereinkommen der Vereinten Nationen zur Bekämpfung der Finanzierung des Terrorismus zu ratifizieren und dessen umfassende Umsetzung sicherzustellen ${ }^{26}$.

Die US-amerikanischen Behörden sowie die Federal Reserve Bank of New York veröffentlichten nach den Anschlägen vom 11. September Listen mit den Namen verdächtiger Personen und Organisationen. In der Schweiz wurden 95 verdächtige Fälle (in welche auf diesen Listen genannte Personen verwickelt waren) der Meldestelle für Geldwäscherei gemeldet. Diese leitete die Dossiers an die Bundesstaatsanwaltschaft weiter, welche untersucht, ob der Verdacht begründet ist. In diesem Kontext bewies die Schweiz ihre Entschlossenheit, den Missbrauch des Finanzplatzes Schweiz für Transaktionen im Zusammenhang mit terroristischen Aktivitäten zu verhindern. Im Herbst 2001 wurden in der Schweiz 72 Konten mit Guthaben von insgesamt 34 Millionen Franken blockiert. Die Untersuchungen ergaben, dass es keine Anhaltspunkte dafür gibt, wonach Personen oder Unternehmen aus der Schweiz an den logistischen Vorbereitungen für die Anschläge in den Vereinigten Staaten beteiligt waren.

24 Informelles System für den Transfer von Vermögenswerten ausserhalb des Bankenwesens, das in Asien verbreitet ist. Eine Person kann Geld bei einem Vertreter des Hawala-Netzwerkes deponieren und erhält dafür eine Art Quittung. Diese kann bei einem anderen Vertreter des Netzwerks gegen Bargeld eingetauscht werden. Die Zahlungen werden zwischen den Vertretern aufgerechnet. Bei dieser Methode hinterlässt der Vermögenstransfer keinerlei Spuren.

25 GAFI, Directives à l'attention des institutions financières pour la détection des activités de financement du terrorisme, Paris, GAFI, 24. April 2002.

26 Der Wortlaut des Übereinkommens ist auf folgender Website verfügbar : <http://untreaty.un.org/English/ Terrorism.asp>. 


\section{$\square$ Bekämpfung der Terrorismusfinanzierung}

Ende Juni 2002 legte Bundesrätin Ruth Metzler einen Entwurf für eine Änderung des schweizerischen Strafgesetzbuches vor, die es erlauben sollte, den Kampf gegen den Terrorismus zu verstärken. Der Entwurf umfasst die Ratifizierung des internationalen Übereinkommens der Vereinten Nationen zur Bekämpfung der Finanzierung des Terrorismus vom 9. Dezember 1999 und des Übereinkommens zur Bekämpfung terroristischer Bombenanschläge aus dem Jahr 1997. Das Übereinkommen über die Bekämpfung der Terrorismusfinanzierung war von der Schweiz am 13. Juni 2001 unterzeichnet worden. Darüber hinaus ist die Schweiz zehn anderen im Rahmen der UNO erarbeiteten Übereinkommen und Protokollen zur Bekämpfung des Terrorismus beigetreten.

Die Mehrzahl der Bestimmungen des Übereinkommens zur Bekämpfung der Terrorismusfinanzierung erfordern keine Anpassungen des Strafrechts, da sie bereits durch die Regelungen betreffend das organisierte Verbrechen (Art. 260ter des Strafgesetzbuches), die Geldwäscherei oder die Korruption abgedeckt sind, so etwa die Bestimmungen des Übereinkommens über das Einfrieren und die Beschlagnahmung von Vermögenswerten mit terroristischem Hintergrund, über die Rechtshilfe, über die Sorgfaltspflichten bei der Identifizierung der Kunden von Finanzinstituten und über die Meldepflicht bei verdächtigen Transaktionen.

Gewisse Änderungen im schweizerischen Strafrecht sind nach Ansicht des Bundesrates dennoch erforderlich. Die wichtigste Änderung besteht darin, dass nicht nur Einzelpersonen und Mitarbeitende (natürliche Personen), sondern auch Unternehmen für das Delikt der Terrorismusfinanzierung bestraft werden können (Verantwortlichkeit juristischer Personen). Anlässlich der Debatte über das Geldwäschereigesetz (1998) und der Änderung des Strafgesetzbuches im Zusammenhang mit der Bestechung ausländischer Amtsträger (2000) hatte das Parlament die Möglichkeit der Bestrafung juristischer Personen abgelehnt mit der Begründung, eine solche Bestimmung sei im Rahmen einer Änderung des allgemeinen Teils des Strafgesetzbuches zu erwägen. Der Bundesrat hatte eine subsidiäre Verantwortlichkeit von Unternehmen bei Organisationsmängeln vorgeschlagen ${ }^{27}$. Das Parlament hingegen befürwortete eine weiter gehende Regelung und sah eine primäre Verantwortlichkeit von Unternehmen für die Delikte der kriminellen Organisationen (Art. 260ter), der Geldwäscherei (Art. 305bis) und verschiedener Bestechungstatbestände (Art. 322ter, 322quinquies und 322septies) vor, bei welchen das Unternehmen für Kontrollmängel verantwortlich gemacht wird. Artikel 5 des Übereinkommens verlangt von den Vertragsstaaten, dass juristische Personen für die Finanzierung terroristischer Handlungen zur Verantwortung gezogen werden können. In der Botschaft wird die Verantwortlichkeit von juristischen Personen erneut aufgegriffen, denn der Bundesrat wollte das internationale Übereinkommen noch vor Ende 2002 ratifizieren, und der Revisionsentwurf des allgemeinen Teils des Strafgesetzbuches hätte vom Parlament bis dahin nicht abschliessend debattiert werden können.

27 Botschaft vom 21. September 1998 zur Änderung des Schweizerischen Strafgesetzbuches (Allgemeine Bestimmungen), BB1 1999, 1979, S. 2136 ff. 


\section{Vom Bundesrat vorgeschlagene Änderung des Strafgesetzbuches zur Bestrafung der Terrorismusfinanzierung}

Art. 100quater

ـ „Wird in einem Unternehmen in Ausübung geschäftlicher Verrichtungen im Rahmen des Unternehmenszwecks ein Verbrechen oder Vergehen verübt und kann diese Tat wegen mangelhafter Organisation des Unternehmens keiner bestimmten natürlichen Person zugerechnet werden, so wird das Verbrechen oder Vergehen dem Unternehmen zugerechnet. In diesem Fall wird das Unternehmen mit Busse bis zu 5 Millionen Franken bestraft."

- Handelt es sich um gewisse schwere Straftaten, „so wird das Unternehmen unabhängig von der Strafbarkeit natürlicher Personen bestraft, wenn dem Unternehmen vorzuwerfen ist, dass es nicht alle erforderlichen und zumutbaren organisatorischen Vorkehren [sic] getroffen hat, um eine solche Straftat zu verhindern".

Der Bundesrat schlägt vor, die Bestimmungen des Artikels 260ter über die Bekämpfung des organisierten Verbrechens auf strafbare Handlungen bei der Vorbereitung terroristischer Aktivitäten auszudehnen. Artikel 260ter ist bereits heute nicht nur auf mafiose Tätigkeiten zugeschnitten, sondern auch auf weitere Handlungen anwendbar, darunter auch auf den Terrorismus (Art. 260bis). Terrorismus wird nach dem neuen Artikel 260quinquies und Finanzierung des Terrorismus nach dem neuen Artikel 260sexies bestraft, wobei zwischen der Finanzierung und dem Verbrechen selbst nicht zwingend ein Kausalzusammenhang bestehen muss. Wer terroristische Handlungen finanziert, ist nur strafbar, sofern es seine Absicht war, Terrorakte zu finanzieren. Wer für wohltätige Zwecke Geld spendet, das indirekt und gegen seinen Willen zur Finanzierung von Terrorakten verwendet wird, ist nicht strafbar.

In ihrer ersten Reaktion auf die Botschaft des Bundesrates vertraten gewisse Beobachter die Ansicht, das bestehende gesetzgeberische Instrumentarium reiche aus, um den Terrorismus bekämpfen zu können, und es bestehe keine Notwendigkeit für neue strafrechtliche Normen. Bereits heute sei die Bestrafung einer Person für Handlungen im Zusammenhang mit dem organisierten Verbrechen (Art. 260ter) quasi unmöglich (seit 1994 wurde diese Strafnorm im Kanton Zürich bereits mehr als hundert Mal geltend gemacht, aber es kam noch nie zu einer Verurteilung auf der Grundlage dieses Artikels) ${ }^{28}$.

Am 23. September 2002 beschloss der Ständerat mit 27 gegen 15 Stimmen die Rücküberweisung der Vorlage an die zuständige Kommission des Ständerates zur neuerlichen Überarbeitung. Dick Marty (FDP/TI) schlug vor, die beiden internationalen Übereinkommen unverzüglich $\mathrm{zu}$ ratifizieren und die Debatte über die Änderungen des schweizerischen Strafgesetzes zu vertagen. In Bezug auf die zur Erfüllung der Bestimmungen der beiden Übereinkommen erforderlichen Änderungen waren die Experten geteilter Meinung. Schliesslich befürworteten sie mehrheitlich die Rücküberweisung der gesamten Vorlage an die Kommission. Am 2. Dezember 2002 befasste sich der Ständerat erneut mit dem Geschäft und hiess die Ratifizierung der beiden internationalen Übereinkommen gut. Die kleine Kammer folgte dem Antrag der Kommission, auf die Verab-

28 Vgl. dazu den Artikel von Josef Estermann, NZZ, 30. August 2002. 
schiedung einer eigentlichen Terrorismusstrafnorm $\mathrm{zu}$ verzichten, und beschränkte sich darauf, die Finanzierung terroristischer Handlungen unter Strafe zu stellen.

\subsection{FINANZPLATZ SCHWEIZ, BANKGEHEIMNIS UND STEUERFLUCHT}

Mehrere Studien haben die schädigenden Auswirkungen der Kapitalflucht auf die Wirtschaft der Entwicklungsländer aufgezeigt. Laut einer Schätzung der NGO Oxfam verursacht die Kapitalflucht in den Entwicklungsländern Steuerausfälle von rund 50 Milliarden Dollar pro Jahr. Dies entspricht in etwa dem Umfang der von den Mitgliedsländern des OECD-Entwicklungshilfeausschusses (DAC) geleisteten öffentlichen Entwicklungshilfe ${ }^{29}$.

Gemäss der heutigen schweizerischen Gesetzgebung wird Steuerbetrug (Urkundenfälschung zur Umgehung der Besteuerung) strafrechtlich geahndet, nicht aber die einfache Steuerhinterziehung (gewisse Einkünfte werden nicht deklariert). Verschiedene Vertreter der Strafverfolgungsbehörden, darunter Bernard Bertossa, halten diese Unterscheidung für problematisch und vertreten die Ansicht, die Schweiz müsse auch bei der Bekämpfung der Steuerflucht den Behörden eines anderen Staates Rechtshilfe gewähren. Kriminelle Organisationen, Auftraggeber von terroristischen Handlungen sowie Personen, die Vermögenswerte dem Zugriff der Steuerbehörde entziehen, bedienen sich bei ihren Machenschaften sehr ähnlicher finanzieller Instrumente, so dass bei der Untersuchung verdächtiger Finanztransaktionen eine Unterscheidung dieser Kategorien schwierig ist.

Gesamthaft betrachtet bietet die schweizerische Gesetzgebung in Bezug auf Geldwäscherei, Korruption und organisiertes Verbrechen recht umfangreiche Rechtshilfemöglichkeiten. Allerdings gewährt die Schweiz nach dem Grundsatz der zweifachen Einstufung als Straftat (wonach der Straftatbestand in beiden beteiligten Ländern gegeben sein muss) in Fällen von Steuerflucht keine Rechtshilfe, da diese in der Schweiz nicht strafbar ist. Die Möglichkeiten zur internationalen Amtshilfe hingegen sind in der Schweiz ziemlich begrenz $\mathrm{t}^{30}$. Sie umfassen beispielsweise die Zusammenarbeit der Eidgenössischen Bankenkommission mit ausländischen Aufsichtsorganen (Marktaufsicht gemäss Banken-, Börsen- und Anlagefondsgesetz). Die ausländische Aufsichtsbehörde darf vertrauliche Informationen nur im Zusammenhang mit dem fraglichen Fall verwenden. Auch die Kontrollstelle und die Meldestelle für Geldwäscherei können einschlägige Informationen an gleichgestellte ausländische Behörden weitergeben. In Zollfragen (Bekämpfung des Zollbetrugs) hingegen sind die Möglichkeiten zur Amtshilfe relativ begrenzt und beschränken sich auf Fragen des Steuerbetrugs.

\section{$\square$ Druck seitens der OECD und der Europäischen Union}

Der von der OECD und der Europäischen Union ausgehende Druck auf die Schweiz, das Bankgeheimnis zu lockern und die Steuerflucht strafrechtlich zu verfolgen, nimmt laufend zu. 1998 hatte die OECD einen Bericht über den schäd-

29 Oxfam, Tax Havens : Releasing the Hidden Billions for Poverty Eradication, Grossbritannien, Oxfam, 2000 (verfügbar auf folgender Website: <www.oxfam.org >).

30 Zwischen den Vereinigten Staaten und der Schweiz ist die Amtshilfe sehr umfangreich. 
lichen Steuerwettbewerb verabschiedet, in dem ein System für den Informationsaustausch zwischen den Steuerbehörden der OECD-Mitgliedsländer gefordert wurde. Die Schweiz und Luxemburg hatten sich bei der Verabschiedung des Berichts der Stimme enthalten. Im April 2000 veröffentlichte der OECD-Ausschuss für Steuerfragen (Committee on Fiscal Affairs, CFA) einen Bericht, in dem die Länder aufgefordert wurden, die Hürden beim Zugang der Steuerbehörden zu gewissen steuerrechtlich relevanten Bankinformationen zu beseitigen (Amtshilfe). Die Schweiz erklärte sich bereit, die Möglichkeiten der Amtshilfe in Fällen von Steuerbetrug abzuklären (analog zur bereits bestehenden Regelung zwischen der Schweiz und den USA), nicht aber in Fällen von Steuerflucht.

Die Europäische Union (EU) hingegen arbeitet auf ein noch vollständigeres System hin. Dieses soll den Informationsaustausch zwischen Steuerbehörden gewährleisten im Zusammenhang mit der Besteuerung von Vermögenserträgen, die von einem Einwohner eines EU-Landes in einem anderen EU-Land erzielt werden (nach einer Übergangsfrist von sieben Jahren nach Verabschiedung der entsprechenden Richtlinie). Bevor jedoch die Richtlinie verabschiedet werden kann, muss die EU mit den Vereinigten Staaten, der Schweiz, Liechtenstein, Monaco, Andorra und San Marino entsprechende Verhandlungen führen, damit diese analoge Massnahmen ergreifen. Die Gespräche mit der Schweiz erfolgen im Rahmen der bilateralen Verhandlungen zwischen der Schweiz und der EU. Die Schweiz ist bereit, auf Erträge von Kapitalanlagen ausländischer Personen eine Zahlstellensteuer zu erheben (und die entsprechenden Steuereinnahmen an die EU-Staaten abzuführen), lehnt aber einen systematischen Informationsaustausch zwischen Steuerbehörden kategorisch ab. Auf ein entsprechendes Gesuch hin könnte die Schweiz in Fällen von Steuerbetrug (und vergleichbaren Handlungen ${ }^{31}$ ) die Weitergabe von Informationen gewähren. Handelt es sich jedoch um einfache Steuerhinterziehung, wird auf ein solches Gesuch nicht eingetreten. Bis Ende 2002 ist zwischen der EU und der Schweiz keine Einigung zustande gekommen, und es herrscht eine Pattsituation. Aber auch innerhalb der Europäischen Union sorgt der Richtlinienentwurf für Divergenzen, denn einige Länder wünschen, dass die Regelung auch für die zu Grossbritannien gehörenden Gebiete Geltung besitzt, eine Forderung, die das Vereinigte Königreich ablehnt.

Die Schweiz nimmt in dieser Frage nach wie vor eine defensive Haltung ein. Vertreter der Bankenkreise, die Eidgenössische Finanzverwaltung sowie das Eidgenössische Volkswirtschaftsdepartement unter der Leitung von Bundesrat Pascal Couchepin bekräftigen, das schweizerische Bankgeheimnis sei von grosser Bedeutung und stehe nicht zur Debatte, denn eine Lockerung des Bankgeheimnisses hätte dramatische Auswirkungen auf die Beschäftigten des Bankensektors. Immerhin trägt der Finanzsektor mit 10 Prozent zum schweizerischen BIP bei ${ }^{32}$. Mehr als die Hälfte der Wertschöpfung des Bankensektors stammt aus dem Vermögensverwaltungsgeschäft. 1998 waren im Finanzsektor gesamthaft rund 218'000 Personen beschäftigt, davon 119'000 bei den Banken, 54'000 bei den Versicherungen und 45'000 bei Treuhandgesellschaften und anderen Unternehmen im Finanzdienstleistungssektor. Darüber hinaus sind rund 130'000 Mit-

31 Der von der Schweiz verwendete Begriff lautet „tax fraud and the like“. Die Schweiz könnte auf bilateraler Ebene mit den einzelnen EU-Ländern festlegen, welche Kategorien vor dem Hintergrund der steuerrechtlichen Regelungen des betreffenden Landes dabei zu berücksichtigen sind.

32 Angaben der Eidgenössischen Bankenkommission. 
arbeitende bei ausländischen Zweigstellen schweizerischer Finanzinstitute beschäftigt. Laut Angaben der Schweizerischen Nationalbank werden in der Schweiz Kundendepots in Höhe von rund 3'800 Milliarden Franken verwaltet ${ }^{33}$.

\section{$\square$ Forderungen der Hilfswerke}

Die NGO Oxfam nennt eine Reihe von Ansätzen zur Verringerung der schädigenden Auswirkungen von so genannten Steuerparadiesen:

- Multilaterale Erarbeitung gemeinsamer Besteuerungsstandards, um die Möglichkeiten international tätiger Unternehmen zur Steuerhinterziehung zu verringern ;

๑ Multilaterale Vereinbarung zur global einheitlichen Besteuerung international tätiger Unternehmen;

๖ Schaffung einer weltweiten Steuerbehörde ;

口 Erarbeitung eines internationalen Übereinkommens zur Erleichterung der Rückführung von Geldern, die aus dem Staatshaushalt armer Länder stammen. Durch den Abschluss einer völkerrechtlichen Konvention soll die Identifizierung und Rückerstattung illegal erworbener Vermögenswerte erleichtert werden;

- Code of conduct für die Bezahlung von Steuern (analog zu Vereinbarungen im Umweltrecht oder in Bezug auf arbeitsrechtliche Mindeststandards) ;

- Abschluss einer multilateralen Vereinbarung betreffend den Informationsaustausch zwischen Steuerbehörden verschiedener Länder zur Verstärkung des Kampfes gegen die Steuerflucht.

Die Arbeitsgemeinschaft der Hilfswerke unterstützt diese Vorschläge gesamthaft und stellt darüber hinaus spezifische Forderungen, die die Schweiz betreffen ${ }^{34}$ :

- Die derzeitigen Gespräche mit der Europäischen Union über die Steuerflucht betreffen ausschliesslich Personen im EU-Raum. Die Arbeitsgemeinschaft fordert den Bund auf, eine allfällige neue Regelung zwischen der Schweiz und der EU im Sinne der Meistbegünstigungsklausel auf sämtliche Länder anzuwenden, denn eine strafrechtliche Verfolgung der Steuerflucht aus den Ländern der EU, nicht aber aus anderen Ländern, wäre schlicht inkohärent. Ebenfalls inkohärent wäre eine gegenseitige Besteuerung von Kapitalerträgen zwischen der Schweiz und der EU, ohne dass auch Erträge auf Guthaben von Personen aus Entwicklungsländern besteuert würden.

- Der internationale Informationsaustausch in Steuerfragen wie auch in Bezug auf Geldwäscherei muss verstärkt werden.

$\checkmark$ Die Schweiz soll nicht nur die Steuerhinterziehung, sondern auch die Steuerflucht strafrechtlich verfolgen und in Steuerfragen Amtshilfe gewähren. Verschiedene schweizerische NGO und Justizvertreter aus der ganzen Welt sind der Ansicht, die in der Schweiz gemachte Unterscheidung zwischen Steuerbetrug (strafbar) und Steuerhinterziehung (nicht strafbar) sei schlichtweg unhaltbar.

33 Statistisches Monatsheft der SNB, Juni 2000.

34 Quelle: Von der Arbeitsgemeinschaft der Hilfswerke im Oktober 2001 durchgeführte Tagung in Bern; Bruno Gurtner „Exigences pour une politique de développement moins inéquitable“ in D. Froidevaux, La Suisse dans la constellation des paradis fiscaux. 
๖ Durch die Schaffung einer internationalen Steuerbehörde liessen sich die negativen Auswirkungen verschiedener nationaler Steuersysteme eindämmen.

- Offshore-Tätigkeiten von Schweizer Unternehmen müssen verstärkt beaufsichtigt werden.

- Ferner muss die Schweiz auch die Bestechung zwischen Privatpersonen strafrechtlich verfolgen (derzeit kennt das Strafgesetzbuch lediglich die Bestechung ausländischer Amtsträger als Straftatbestand).

๖ Schliesslich muss die Schweiz abklären, ob die Steuerflucht nicht durch Doppelbesteuerungsabkommen gefördert wird.

In seiner Antwort auf eine Interpellation von Nationalrat Remo Gysin (SP/BS) nahm der Bundesrat zum ersten Punkt Stellung. Aus der Sicht des Bundesrates besteht kein Anlass, eine allfällige Vereinbarung mit der Europäischen Union im Sinne der Meistbegünstigungsklausel auf die Entwicklungsländer auszudehnen ${ }^{35}$. Im internationalen Steuerrecht gebe es keinen Meistbegünstigungsstandard. Aus diesem Grund würde ein allfälliges Abkommen zwischen der Schweiz und der EU seine Wirkung nur zwischen den unterzeichneten Vertragsstaaten entfalten. Die Zahlstellensteuer würde ausschliesslich zu Gunsten der EU-Mitgliedsländer erhoben. Zudem seien von anderer Seite als der EU auch keine entsprechenden Begehren an die Schweiz gerichtet worden.

\section{QUELLEN}

\section{Fachliteratur}

Dominique Froidevaux (sous la direction de), La Suisse dans la constellation des paradis fiscaux, Lausanne, Editions d'en Bas, 2002, 240 S.

Christian J. Meier-Schatz, Peter Nobel, Robert Waldburger, Die Auswirkungen eines EU-Beitritts auf den Finanzplatz Schweiz, Zürich, Schultess, 2001, 280 S.

Anne Mette Skipper, La Suisse, les banques et l'argent sale, Lausanne, Esprit Ouvert, 2001.

Mark Pieth, Mario von Cranach, Claudio Besozzi, Christa Hanatseder, Karl-Ludwig Kunz, Gewalt im Alltag und organisierte Kriminalität. Die Ergebnisse eines nationalen Forschungsprogramms, Bern/Stuttgart, Wien, Verlag Haupt, 2002.

Josef Estermann, Organisierte Kriminalität in der Schweiz, Orlux-Verlag, Postfach 2817, 6002 Luzern, $196 \mathrm{~S}$.

\section{Berichte}

GAFI, Rapport sur les typologies du blanchiment de capitaux 2001-2002, Paris, Februar 2002.

GAFI, Révision des quarante recommandations du GAFI, document consultatif, Paris, GAFI, 30. Mai 2002.

GAFI/FATF, Review to Identify Non-Cooperative Countries or Territories: Increasing The World-Wide Effectiveness of Anti-Money Laundering Measures, Paris, GAFI, 21. Juni 2002.

GAFI/FATF, Annual Report 2001-2002, Paris, 21. Juni 2002.

Bericht zur Finanzsystem-Stabilität des IWF (in englischer Sprache): <www.imf.org $>$ oder $<w w w$. dff.admin.ch>.

Bundesamt für Polizei, Meldestelle für Geldwäscherei (MROS), 4. Rechenschaftsbericht, Mai 2002.

DEZA/SDC, Partners for development and against corruption. The SDC perspective on Swiss financial transparency, DEZA, Bern, 2002.

Eidgenössische Bankenkommission, Sorgfaltspflichten von Banken und Effektenhändlern bei Geldwäscherei, Terrorismusfinanzierung und Beziehungen zu politisch exponierten Personen, Bericht der „Arbeitsgruppe KYC“, Bern, Juni 2002, 40 S.

Eidgenössische Bankenkommission, Regulatory challenges for Swiss banking secrecy, Bern, 21. Juni 2002.

35 Medienmitteilung des Eidgenössischen Finanzdepartements, 29. November 2002. 
Oxfam, Tax Havens: Releasing the Hidden Billions for Poverty Eradication, Grossbritannien, Oxfam, 2000 (verfügbar auf folgender Website: attac.org.uk/html/view-document.um?documentID=44-uk)

Rapport Montebourg: Rapport d'information déposé par la mission d'information commune de l'Assemblée nationale sur les obstacles au contrôle et à la répression de la délinquance financière et du blanchiment des capitaux en Europe - La Suisse (la lutte contre le blanchiment de capitaux en Suisse, un combat de façade), Paris, Editions des Arènes, 2001.

\section{Presseartikel und Medienmitteilungen}

Diverse Artikel der Westschweizer Tageszeitung Le Temps (namentlich die im August 2002 erschienene Artikelserie über die Geschichte des Bankgeheimnisses) und der Neuen Zürcher Zeitung (NZZ).

Le Courrier, 26. November 2002.

Pressemitteilungen der Bundesverwaltung <www.admin.ch $>$.

Medienmitteilungen der Bundesverwaltung über die Verhandlungen zwischen der Europäischen Union und der Schweiz über die Zinsenbesteuerung: Medienmitteilungen vom 18. Juni 2002, 31. Oktober 2002 und 29. November 2002.

\section{INTERNET-ADRESSEN}

OECD-Arbeitsgruppe gegen Geldwäscherei (FATF) : <www.oecd.org/fatf>.

OECD, Site zur Korruption : <www.oecd.org/daf/nocorruption>.

International Anti-Money Laundering Network: <www.imolin.org $>$.

UNO, Anti-Money Laundering Web-Site : <www.imolin.org $>$.

Europäische Union: <www.europa.eu.int>.

Sämtliche schweizerischen Gesetze und Verordnungen sind in der Systematischen Sammlung des Bundesrechts gesammelt und können auf folgender Internet-Adresse konsultiert werden: <www. admin.ch/ch/d/sr> (siehe vor allem Ziffer 955, Geldwäscherei)

Eidgenössisches Justiz- und Polizeidepartement: <www.ejpd.admin.ch>.

Meldestelle für Geldwäscherei (Bundesamt für Polizei) : <www.admin.ch/bap >.

Eidgenössische Bankenkommission: <www.admin.ch/ebk>.

Eidgenössische Finanzverwaltung/Kontrollstelle für die Bekämpfung der Geldwäscherei : <www.admin. ch/efv $>$.

\section{Nichtregierungsorganisationen :}

Transparency International: <www.transparency.org $>$.

Erklärung von Bern: <www.evb.ch $>$.

Aktion Finanzplatz Schweiz, Drahtzugstr. 28, 4057 Basel (Herausgeberin des Informationsbulletins Finanzplatz Informationen). 